\title{
Communication Skills of Grandview/Southview Medical Center General Surgery Residents
}

Wesley Johnson, DO; Nhat-Anh Ngo, DO; Michael Elrod, DO

From the Grandview Medical

Center in Dayton, Ohio, and the Ohio University Heritage College of Osteopathic Medicine in Athens, Ohio. Financial Disclosures: None reported.

Support: None reported.

Address correspondence to

Wesley Johnson, DO, Grandview Medical Center, 405 W Grand Ave, Dayton, Ohio 45405-4796.

Email:wj494911@ohio.edu

Submitted August 25, 2019; revision received January 3, 2020; accepted January $27,2020$.
Context: In the transition of osteopathic programs to the single-accreditation graduate medical education (GME) system, residents are required to demonstrate skill in a set of core competencies identified by the Accreditation Council of Graduate Medical Education (ACGME) prior to graduation. Included in those core competencies are interpersonal and communication skills along with professionalism.

Objectives: To assess strengths and weaknesses of residents' interpersonal communication skills and professionalism in the Grandview/Southview Medical Center (Dayton, OH) osteopathic general surgery program using the validated Communication Assessment Tool (CAT).

Methods: From November 2014 to June 2018, all patients who presented for an appointment at the Cassano General Surgery Clinic were asked by a medical assistant to complete a CAT questionnaire following their encounter with a resident physician. Patients at Cassano, an outpatient office-based facility directed to the underserved local community, are seen first by an intern, then by a $4^{\text {th }}$ or $5^{\text {th }}$ year resident and later by an attending physician. Patients 18 years of age or older were included; patients were excluded if they were unable to understand or read English. Patient demographics were collected, including age, gender, race/ethnicity, and previous exposure to this resident physician. Each resident's name was replaced on the CAT with a number for data analysis. The resident variables collected for this study included year of training, gender, and native language.

Results: The mean response for all CAT items was 4.5 out of 5 , indicating that responses to resident performance were largely positive. Patients responded to 4 of the 14 CAT items with only excellent, very good, or good responses and no fair or poor responses. Four items had only 1 fair or poor response. The remaining 6 items received more than 1 fair or poor response: "greeted me in a way that made me feel comfortable" (\#1), "talked in terms I could understand" (\#8), "encouraged me to ask questions" (\#10), "involved me in decisions as much as I wanted" (\#11), "showed care and concern" (\#13), and "spent the right amount of time with me" (\#14).

Conclusions: Attending surgeons evaluate residents in multiple areas from a doctor's perspective, but there is a potential lack of correlation between that evaluation and a patient's experience, which is paramount in osteopathic medicine. Patient responses to the CAT questionnaire can be used by program directors to identify deficiencies in milestone/competency achievement and facilitate improvement both individually and programmatically for residents according to ACGME standards.

J Am Osteopath Assoc. 2020;120(12):865-870. Published online October 29, 2020 doi: 10.7556/jaoa.2020.122

Keywords: ACGME, communication, competency, medical education, resident, surgery 
$\mathrm{T}$ he 4 primary tenets of osteopathic medicine translate well to surgical care. First, the body's innate ability to heal allows surgical manipulation, incision, and excision of structures to promote function. In the perioperative period, including in the outpatient setting, osteopathic physicians attempt to treat the patient's mind, body, and spirit. Thinking about how pathology affects the entire patient, instead of just a patient's individual components, leads to the best care. With these ideas in mind, the osteopathic general surgery program at Grandview/Southview Medical Center in Dayton, Ohio, continues to assess its strengths and deficiencies for the sake of quality improvement, both in resident education and patient care. One component of this assessment focuses on resident performance in the Cassano General Surgery Clinic, an outpatient office-based facility. This service is directed to the underserved local community, managed by residents, and overseen by attending physicians. Patients who present to the facility are evaluated first by an intern, then by a fourth or fifth-year resident, and subsequently, an attending physician.

The Accreditation Council of Graduate Medical Education (ACGME) has instituted a method to assess resident progression through a given program. Following the transition of osteopathic programs to the single-accreditation graduate medical education (GME) system, residents are required to demonstrate skill in a set of core competencies identified by the ACGME before graduation. Skill is determined by achieving milestones in each competency, ensuring continued improvement throughout 5 years of training. ${ }^{1}$ These core competencies translate well to previously established osteopathic principles; we continue to focus on treating the patient as an overall functional unit.

The ACGME milestones of interpersonal communication skills and professionalism are measured through the Communication Assessment Tool (CAT), which is a paper-based survey of patient perceptions of care (Figure 1) originally developed and validated by Makoul et al. ${ }^{2}$ The CAT was subsequently validated by other researchers, including Myerholtz et $\mathrm{al}^{3}{ }^{3}$ who used it first to assess family medicine residencies in Toledo, Ohio at Mercy Saint Vincent Hospital. ${ }^{3}$ The CAT contains 14-questions rating physician performance on a 5-point Likert-type scale, poor (1) to excellent (5). In addition, resident and patient demographics are recorded and evaluated.

In this study, we report the results of our evaluation of resident communication skills and professionalism in the outpatient setting using the CAT.

\section{Methods}

From November 2014 to June 2018, all patients who presented for an appointment at the Cassano General Surgery Clinic were asked by a medical assistant (MA) to complete a CAT questionnaire following their encounter with a resident physician. Patients 18 years of age or older were included; patients were excluded if they were unable to understand or read English. The MA informed patients that completion of the survey was voluntary and that responses were confidential. Patient demographics were collected, including age, gender, race/ethnicity, and previous exposure to this resident physician. After the patient returned the completed CAT form to the MA, it was placed in a sealed envelope. At no point were residents allowed to participate in requests to complete the CAT or in collecting CAT surveys. The resident variables collected for this study included year of training, gender, and native language. Although it was made clear to each patient by the MA's verbal description which resident was being evaluated through the CAT, names were replaced with numbers for data analysis.

Expedited approval for this prospective study was given by the institutional review board through the Kettering Health Network.

The primary investigator at the time (A.N.) collected CAT surveys for data aggregation and analysis. Data were entered into Microsoft Excel and imported into IBM SPSS Statistics for Windows, Version 24.0, (IBM Corp.) for analysis. Nominal variables were summarized with frequencies and percentages. Continuous 


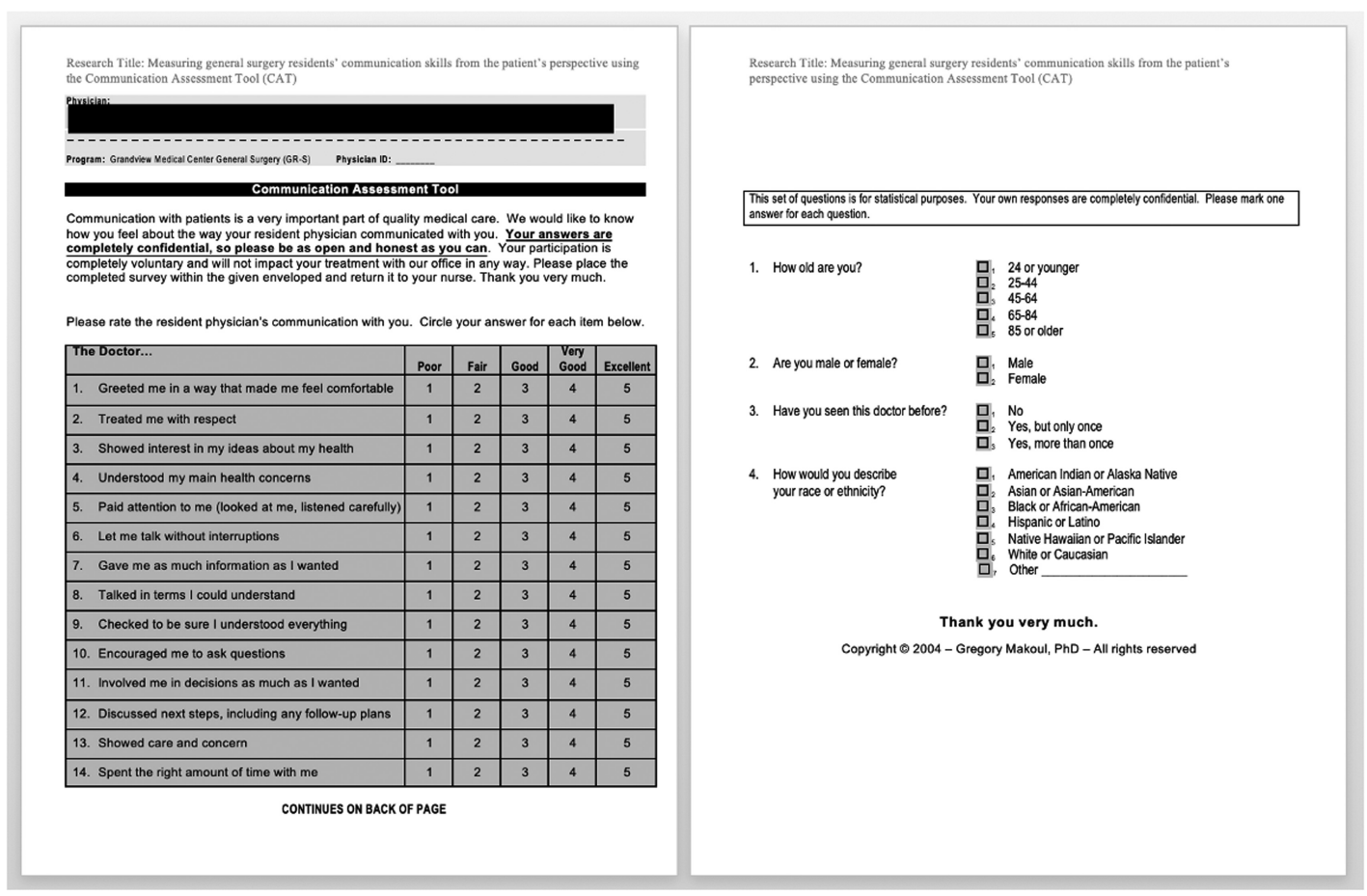

Figure 1.

Communication Assessment Tool used in this study, which included a resident evaluation questionnaire on the front and patient demographics on the back.

variables were summarized with mean, standard deviation, median, and range.

\section{Results}

A total of 18 residents rotated through the Cassano General Surgery Clinic during the study period (13 men, $72 \%$; 5 women, 28\%). While there are usually 11 to 12 residents in the program at any given year, with 2 to 3 graduating yearly and 2 to 3 joining the program, some residents had already graduated when this study began in 2014; therefore, the total number was 18 . All residents except 1 (17; 94\%) were native English speakers who identified as white/Caucasian. The 1 nonnative English speaker was of Asian descent.

Patient characteristics are presented in Table $\mathbf{1}$. A total of 220 patient responses were collected. The most common patient age group was 45 to 64 years (101; $46 \%) ; 38 \%$ of patients (86) were under 45 years of age, and 15\% (16) were 65 or older. Most patients $(128 ; 58 \%)$ were women, and most reported not having seen the current resident physician before. The most common race/ethnicity reported by patients was white or Caucasian (106; 48\%), followed by Black or African American $(71 ; 32 \%)$; approximately $8 \%$ of patients (17) chose not to specify a race.

The mean response for all CAT items was 4.5 out of 5 ; medians were all 5 out of 5 , indicating that responses that were skewed to favor resident performance (Figure 2), given that patients tend to answer positively when evaluating others. A total of $62.79 \%$ of responses to resident performance were excellent. Patients responded to 4 of the 14 CAT items (28.5\%) with only excellent, very good, or good responses and no fair or poor responses (Table 2). These items included "treating the patient with respect" (\#2), "paying attention" (\#5), "not interrupting" (\#6), and "checking for understanding" (\#9). Four items (28.5\%) had only 1 fair or 
Table 1.

Patient Characteristics $(\mathbf{n = 2 2 0})$

\begin{tabular}{|c|c|}
\hline ariable & $\mathbf{N}(\%)$ \\
\hline \multicolumn{2}{|l|}{ Age, years } \\
\hline$\leq 24$ & $14(5.4)$ \\
\hline $25-44$ & $72(32.7)$ \\
\hline $45-64$ & $101(45.9)$ \\
\hline $65-84$ & $16(7.3)$ \\
\hline Missing & $17(7.7)$ \\
\hline \multicolumn{2}{|l|}{ Sex } \\
\hline Male & $75(34.1)$ \\
\hline Female & $127(57.7)$ \\
\hline Missing & $18(8.2)$ \\
\hline \multicolumn{2}{|l|}{ Has seen this doctor before } \\
\hline No & $122(55.5)$ \\
\hline Yes, but only once & $36(16.4)$ \\
\hline Yes, more than once & $40(18.2)$ \\
\hline Missing & $22(10.0)$ \\
\hline \multicolumn{2}{|l|}{ Race/Ethnicity } \\
\hline American Indian or Alaska Native & $6(2.7)$ \\
\hline Black or African American & $71(32.3)$ \\
\hline Hispanic or Latino & $11(5.0)$ \\
\hline Native Hawaiian or Pacific Islander & $1(0.5)$ \\
\hline White or Caucasian & $106(48.2)$ \\
\hline Other & $8(3.6)$ \\
\hline Missing & $17(7.7)$ \\
\hline
\end{tabular}

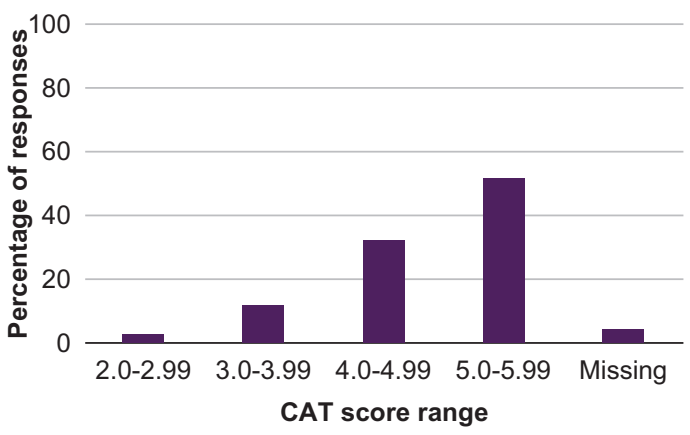

Figure 2.

Overall Communication Assessment Tool (CAT) mean. poor response. These items included "showed interest in my ideas about my health" (\#3), "understood my main health concern" (\#4), "gave me as much information as I wanted" (\#7), and "discussed next steps, including any follow up plans" (\#12). Six items (37.5\%) had more than 1 fair or poor response: "greeted me in a way that made me feel comfortable" (\#1), "talked in terms I could understand" (\#8), "encouraged me to ask questions" (\#10), "involved me in decisions as much as I wanted" (\#11), "showed care and concern" (\#13), and "spent the right amount of time with me" (\#14).

\section{Discussion}

Attending surgeons evaluate residents in multiple areas from a doctor's perspective. However, evaluation results may vary greatly from a patient's perspective; previous authors, including Resnick et $\mathrm{al},{ }^{4}$ have suggested a lack of correlation between these 2 entities in resident evaluation. The key to osteopathic medicine is treating each patient as a whole, making their perspective paramount. Given our definitions of the variables in this study, the results yielded a left (negative) skew. Favoring human performance in a survey is a relatively common phenomenon. One could view the data as difficult to interpret because of the skew in favor of resident performance. Conversely, the negative responses could be more meaningful as a result. If a patient felt obligated to give a positive response but still recorded a negative one, then it is more likely that resident performance was indeed lacking during that interaction. Our program director had access to each data number assigned to the residents in this study; therefore, concerns regarding ACGME milestone performance could be addressed and resolved prior to graduation, thus making the resident more competent in interpersonal and communication skills and professionalism.

The tendency of patients to view resident performance positively as a potential bias has been observed in other research articles using the CAT survey, including the original study by the creators of the survey. ${ }^{2}$ 


\begin{tabular}{|c|c|c|c|c|c|}
\hline Item & Poor, n (\%) & Fair, $\mathbf{n}(\%)$ & Good, n (\%) & Very Good, n (\%) & Excellent, $n$ (\%) \\
\hline 1. Greeted me in a way that made me feel comfortable & $1(0.5)$ & $3(1.4)$ & $25(11.4)$ & $51(23.2)$ & $140(63.6)$ \\
\hline 2. Treated me with respect & 0 & 0 & $23(10.5)$ & $49(22.3)$ & $148(67.3)$ \\
\hline 3. Showed interest in my ideas about my health & 0 & $1(0.5)$ & $23(10.5)$ & $57(25.9)$ & $139(63.2)$ \\
\hline 4. Understood my main health concerns & 0 & $1(0.5)$ & $22(10.0)$ & $54(24.5)$ & $143(65.0)$ \\
\hline 5. Paid attention to me (looked at me, listened carefully) & 0 & 0 & $21(9.5)$ & $50(22.7)$ & $149(67.7)$ \\
\hline 6. Let me talk without interruptions & 0 & 0 & $20(9.4)$ & $55(25.9)$ & $137(64.6)$ \\
\hline 7. Gave me as much information as I wanted & 0 & $1(0.5)$ & $26(12.3)$ & $49(23.1)$ & $136(64.2)$ \\
\hline 8. Talked in terms I could understand & $1(0.5)$ & $3(1.4)$ & $16(7.5)$ & $51(24.1)$ & $141(66.5)$ \\
\hline 9. Checked to be sure I understood everything & 0 & 0 & $21(9.9)$ & $54(25.5)$ & $137(64.6)$ \\
\hline 10. Encouraged me to ask questions & $1(0.5)$ & $1(0.5)$ & $30(14.2)$ & $51(24.1)$ & $129(60.8)$ \\
\hline 11. Involved me in decisions as much as I wanted & $1(0.5)$ & $1(0.5)$ & $25(11.8)$ & $53(25.0)$ & $132(62.3)$ \\
\hline 12. Discussed next steps, including any follow-up plans & $1(0.5)$ & 0 & $20(9.4)$ & $56(26.4)$ & $135(63.7)$ \\
\hline 13. Showed care and concern & 0 & $2(0.9)$ & $20(9.4)$ & $52(24.5)$ & $138(65.1)$ \\
\hline 14. Spent the right amount of time with me & 0 & $2(0.9)$ & $24(11.3)$ & $56(26.4)$ & $130(61.3)$ \\
\hline
\end{tabular}

Their recommendation was therefore to only count the excellent (5) responses as being positive, making a clear delineation in performance. Stausmire et al performed a similar study ${ }^{5}$ to ours at Mercy Saint Vincent Hospital (Toledo, Ohio) in the osteopathic and allopathic general surgery program and focused on excellent responses. Their mean of all questions with an excellent response was $70.2 \%{ }^{5}$ These results are very similar to our own, as our "excellent" responses totaled $62.79 \%$. Neither study demonstrated a performance difference based on resident sex or prior patient experience with that particular resident. Neither study was able to evaluate resident performance by year to compare $1^{\text {st }}$ year with $4^{\text {th }}$ or $5^{\text {th }}$ year residents, although previous studies at Mercy Saint Vincent did demonstrate a decrease in performance from program year (PGY) 1 to PGY 2 and 3 in a family medicine program. ${ }^{3}$

Another limitation of our study is the effect that multiple providers have on patient perceptions of care. In our study, the patients interacted with receptionist and
MA before a physician entered the room. Then, an intern, senior resident, and attending physician each interacted with patient. These exchanges likely influenced the evaluation of the resident in question, regardless of his/her performance. Similar concerns have been expressed in other studies, including 1 completed in 2018 by McKinley et al, ${ }^{6}$ which was a survey of resident hospital performance given to postoperative general surgery patients.

Our study could be expanded to address other competencies in the ACGME curriculum. To further evaluate interpersonal and communication skills and professionalism, the study could be expanded to the inpatient setting. This was attempted by Ferranti et $\mathrm{al}^{7}$ using the CAT; in that study, an inpatient hospitalist and teaching service were evaluated, but scores were significantly lower than expected. Surprisingly, many patients could not even recognize the evaluated physician by photo or name. It is unclear whether this was due to the stress of being ill in the hospital, the high 
number of physicians who evaluated each patient (intern, senior resident, attending), or if an insufficient amount of time was spent in the room. It would be interesting to see how this evaluation would compare to a general surgery service.

The CAT evaluation of resident performance is only 1 potential component of continued assessment to ensure future physician competency. Complications in the operating room could be tracked to assess patient care and procedural skills, as well as practice-based learning and improvement. Academic performance could be assessed through formal testing of the residents, thus promoting medical knowledge. Changes to the academic curriculum could then be instituted to improve in-service and board examination scores. Our institution, in compliance with ACGME standards and osteopathic principles, continues attending evaluation of each resident to ensure all criteria are being met appropriately. Each attending evaluates each resident monthly and then each resident is evaluated by the program director quarterly.

\section{Conclusion}

The ACGME strives to ensure surgeon ability and patient care through identification and assessment of milestones in given competencies. These ACGME core competencies parallel the osteopathic principles established throughout the education of every DO surgeon. Our study evaluated resident interpersonal and communication skills and professionalism in an outpatient setting through the CAT. The results were negatively skewed in favor of resident performance, a common survey phenomenon, but poor responses were also recorded. Similar assessments can be used by program directors to identify deficiencies in milestone/competency achievement and facilitate improvement both individually and programmatically. Tracking achievement in the ACGME curriculum can be expanded to other skills in different competencies as well.

\section{Acknowledgments}

The authors would like to thank Stephanie Balsom, Clinical Research Coordinator, for proofreading this manuscript and providing submission recommendations, and Kathleen Mathieson, $\mathrm{PhD}, \mathrm{CIP}$, for providing statistical analysis.

\section{Author Contributions}

Drs Ngo and Johnson provided substantial contributions to conception and design, acquisition of data, or analysis and interpretation of data; Dr Johnson drafted the article and $\mathrm{Dr}$ Elrod revised it critically for important intellectual content; all authors gave final approval of the version of the article to be published; and all authors agree to be accountable for all aspects of the work in ensuring that questions related to the accuracy or integrity of any part of the work are appropriately investigated and resolved.

\section{References}

1. The General Surgery Milestone Project: a joint initiative of the Accreditation Council for Graduate Medical Education and American Board of Surgery. Accreditation Council for Graduate Medical Education website. July 2015. Accessed August 11, 2020. http://www.acgme.org/ portals/0/pdfs/milestones/surgerymilestones.pdf.

2. Makoul G, Krupat $\mathrm{E}$, Chang $\mathrm{CH}$. Measuring patient views of physician communication skills: development and testing of the communication assessment tool. Patient Educ Counsel. 2007; 67(3):333-42. doi:10.1016/j.pec.2007.05.005

3. Myerholtz L, Simons L, Felix S, et al. Using the communication assessment tool in family medicine residency programs. Fam Med. 2010;42:567-73.

4. Resnick A, Disbot M, Wurster A, Mullen J, Kaiser L, Morris J. Contributions of surgical residents to patient satisfaction: impact of residents beyond clinical care. J Surg Educ. 2007;64(2):72. doi: 10.1016/j.jsurg.2008.01.005

5. Stausmire JM, Cashen CP, Myerholtz L, Buderer N. Measuring general surgery residents' communication skills from the patient's perspective using the communication assessment tool (cat). J Surg Educ. 2015;72 (1):108-116. doi:10.1016/j.jsurg.2014.06.021

6. McKinley SK, Wojcik BM, Kochis M, et al. A pilot study of inpatient satisfaction rating of surgical resident care. J Surg Educ. 2018;75(6): e192-e203. doi:10.1016/J.JSURG.2018.08.007

7. Ferranti DE, Makoul G, Forth VE, Rauworth J, Lee J, Williams MV. Assessing patient perceptions of hospitalist communication skills using the communication assessment tool (cat). J Hosp Med. 2010;5 (9):522-527. doi:10.1002/jhm.787

๑) 2020 American Osteopathic Association 